\title{
Reação de genótipos do gênero Psidium spp. a Meloidogyne enterolobii
}

\author{
Edilton de Albuquerque Cavalcanti Junior ${ }^{1,2} \oplus^{\circ}$, Rômulo Maciel de Moraes Filho ${ }^{1,2} \mathbb{C}$, Jackeline Gadé de Araujo

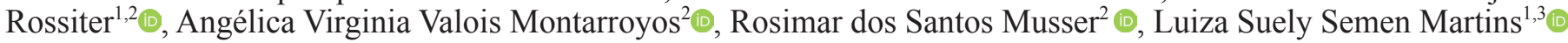

${ }^{1}$ Pós-Graduação da Universidade Federal Rural de Pernambuco, Departamento de Agronomia, UFRPE, Rua Dom Manuel de Medeiros, s/n, Dois Irmãos - CEP: 52171-900, Recife, Pernambuco, Brasil. ${ }^{2}$ Departamento de Agronomia, UFRPE, Rua Dom Manuel de Medeiros, s/n, Dois Irmãos - CEP: 52171-900. Recife, Pernambuco, Brasil. ${ }^{3}$ Departamento de Biologia, UFRPE, Rua Dom Manuel de Medeiros, s/n, Dois Irmãos - CEP: 52171-900, Recife, Pernambuco, Brasil.

Autor para correspondência: Rômulo Maciel de Moraes Filho (romulommfilho@yahoo.com.br)

Data de chegada: 21/03/2018. Aceito para publicação em: 19/12/2019.

$10.1590 / 0100-5405 / 193123$

\section{RESUMO}

Cavalcanti Junior, E.A.; Moraes Filho, R.M.; Rossiter, J.G.A.; Montarroyos, A.V.V.; Musser, R.S.; Martins, L.S.S. Reação de genótipos do gênero Psidium spp. a Meloidogyne enterolobii. Summa Phytopathologica, v.46, n.4, p.333-339, 2020.

A cultura da goiabeira tem sido severamente atacada pelo fitonematóide Meloidogyne enterolobii (Sin.: M. mayaguensis), que tem causado drástica redução de produtividade e, em alguns casos, até morte de todas as árvores do pomar cultivado. Devido à sua ampla gama de potenciais hospedeiros e fácil disseminação, M. enterolobii representa um perigo constante e tem se mostrado um dos maiores limitantes para o cultivo da goiabeira no Nordeste brasileiro. Objetivou-se com esse trabalho avaliar a reação de genótipos de goiabeira e araçazeiro ao parasitismo de M. enterolobii em condições de casa de vegetação.
Foram avaliados 44 genótipos de araçazeiros oriundos de populações naturais do Estado de Pernambuco e seis genótipos de goiabeiras. Os genótipos foram avaliados segundo os seguintes parâmetros: índice de galhas (IG), fator de reprodução (FR), número de ovos por grama de raiz (OGR) e redução do fator de reprodução (RFR). Considerando os critérios avaliados, os genótipos de araçazeiros REC-DI-A02 e REC-DI-A04 alcançaram os melhores resultados, podendo ser indicados como fonte de resistência à $M$. enterolobii e também como genótipos promissores para testes de enxertia em goiabeira.

Palavras-chave: Goiabeira, Myrtaceae, Nematoides das galhas, Resistência.

\section{ABSTRACT}

Cavalcanti Junior, E.A.; Moraes Filho, R.M.; Rossiter, J.G.A.; Montarroyos, A.V.V.; Musser, R.S.; Martins, L.S.S. Reaction of genotypes of the genus Psidium spp. to Meloidogyne enterolobii. Summa Phytopathologica, v.46, n.4, p.333-339, 2020.

The guava crop has been severely attacked by the phytonematode Meloidogyne enterolobii, which has caused drastic reduction in productivity and, in some cases, even the death of all trees in the cultivated orchard. Due to its wide range of potential hosts and easy dissemination, $M$. enterolobii represents a constant threat and has established itself as one of the major constraints for guava cultivation in Northeast Brazil. The objective of this study was to evaluate the reaction of guava and "araçá" genotypes to $M$. enterolobii parasitism under greenhouse conditions. Forty-four "araçá" genotypes from natural populations in Pernambuco State were evaluated, as well as six guava genotypes. The genotypes were evaluated according to the following parameters: gall index (GI), reproduction factor (RF), number of eggs per gram of root (OGR) and reproductive factor reduction (RFR). Considering the evaluated criteria, the "araçá" genotypes REC-DI-A02 and REC-DI-A04 had the best results and can be recommended as a source of resistance to $M$. enterolobii, also constituting promising genotypes for grafting tests in guava.

Keywords: Guava tree, Myrtaceae, Root-knot nematodes, Resistance.

O gênero Psidium possui aproximadamente 150 espécies destacando-se a espécie Psidium guajava L. (goiaba), devido a sua grande importância econômica, e tendo o Brasil como um dos maiores produtores comerciais, com uma área plantada equivalente a 22.269 ha (27). A goiabeira é uma frutífera perene que possui frutos ricos em vitamina $\mathrm{C}$ e sais minerais, bastante apreciados no mercado nacional e internacional (14). A importância econômica do cultivo da goiabeira se dá devido ao elevado valor nutricional de seus frutos e as diversas formas possíveis de consumo, seja in natura, ou como produtos industrializados. Dentre as espécies da família Myrtaceae, a goiabeira e o araçazeiro estão entre as mais importantes economicamente. Apesar do araçazeiro não compartilhar da mesma importância da goiabeira, esta cultura é de grande interesse para pesquisas e para o melhoramento genético devido às características desejáveis de seus frutos, como os elevados teores de vitamina $\mathrm{C}$ e também devido à existência de genótipos resistentes ao fitonematoide Meloidogyne enterolobii YANG e EISENBACK (32) (Sin.: M. mayaguensis). Devido ao fato do parasitismo pelo nematoide predispõe as plantas à podridão da raiz causada por Fusarium solani (Mart.) Sacc, ou seja, a associação sinérgica entre $M$. enterolobii e $F$. solani causa uma doença complexa - o declínio da goiabeira - cujos sintomas são apodrecimento progressivo do sistema radicular, bronzeamento e queima dos bordos das folhas, amarelecimento total da parte aérea e queda das folhas e morte da planta (13). O que tem causado consideráveis danos aos produtores, podendo ser usada como porta-enxerto para as goiabeiras comerciais $(3,28,29)$. O M. enterolobii detectado pela primeira vez na Ilha de Haiman, na 
China em 1983, teve sua presença assinalada nos municípios de Petrolina (PE), Curaça e Maniçoba (BA), recebendo a designação de M. mayaguensis (5). Observa-se também que a ocorrência de M. enterolobii, não é restrita a este Estado, sendo o nematoide observado em diversos Estados das cinco regiões do país (7, 8, $11,12,31)$. Esta situação é agravada devido à baixa variabilidade genética das variedades de goiabeiras comerciais brasileiras, onde aproximadamente $70 \%$ dos pomares são compostos pela cultivar Paluma, destinados ao consumo industrializado (26).

No atual contexto, $M$. enterolobii representa um perigo constante. A sua rápida disseminação e o seu hábito polifágico, que o habilita a parasitar um grande número de espécies, como plantas ornamentais, fumo, soja, cafeeiro, mamão, acerola, araçá, goiaba, plantas daninhas e diversas hortaliças, o que dificulta o manejo desse patógeno difícil controle $(15,20,23)$.

Pesquisas têm sido realizadas com objetivo de encontrar genótipos da família Myrtaceae resistentes ao parasitismo de $M$. enterolobii sendo que araçazeiros (Psidium spp.) tem sido relatados como fonte de resistência, ao nematoide em questão $(3,22,30)$.

O objetivo deste trabalho foi avaliar a resposta de genótipos de araçazeiros e goiabeiras à $M$. enterolobii, e selecionar genótipos potencialmente resistentes ou tolerantes para serem utilizados como porta-enxertos compatíveis com variedades comerciais de goiabeiras.

\section{MATERIAL E MÉTODOS}

O presente trabalho foi desenvolvido na área de Fitotecnia do Departamento de Agronomia, da Universidade Federal Rural de Pernambuco (UFRPE), com localização a 801'92" S, 3456'74" W, a $6 \mathrm{~m}$ de altitude, onde foram avaliados 44 genótipos de araçá e cinco de goiabeira, provenientes do Estado de Pernambuco, do Rio de Janeiro e uma goiabeira comercial obtida em mercado de frutas (Tabela 1).

A coleta dos genótipos para obtenção das sementes ocorreu entre os meses de janeiro a fevereiro de 2016 nos seguintes municípios de Pernambuco: Cabo de Santo Agostinho, Paudalho, Canhotinho e Região Metropolitana do Recife. Foram coletados de três a quatros frutos fisiologicamente maduros ou em estágio de amadurecimento de cada planta. Todos os frutos coletados foram lavados em água corrente para eliminação de resíduos e tratados com solução de hipoclorito a 5\%, despolpados e retirada as sementes que foram secas a sombra.

A semeadura foi realizada em bandejas de polietileno expandido de $16 \times 8$ células, colocando-se duas sementes por célula, utilizando-se substrato comercial Basaplant ${ }^{\mathbb{R}}$ em 13 de maio de 2016. As bandejas foram levadas para casa de vegetação onde receberam regas diárias, tratos culturais e solução nutritiva formulada na proporção de $20 \mathrm{~g}$ de Sulfato de Magnésio $\left(\mathrm{MgSO}_{4}\right), 37,5 \mathrm{~g}$ de Nitrato de Cálcio $\left(\mathrm{Ca}\left(\mathrm{NO}_{3}\right)_{2}\right)$, 22,5 g de Nitrato de Potássio $\left(\mathrm{KNO}_{3}\right), 1,25 \mathrm{~g}$ de Quelatec A-Z, $10 \mathrm{~g}$ de

Tabela 1. Origem e identificação dos genótipos de Psidium spp. avaliados.

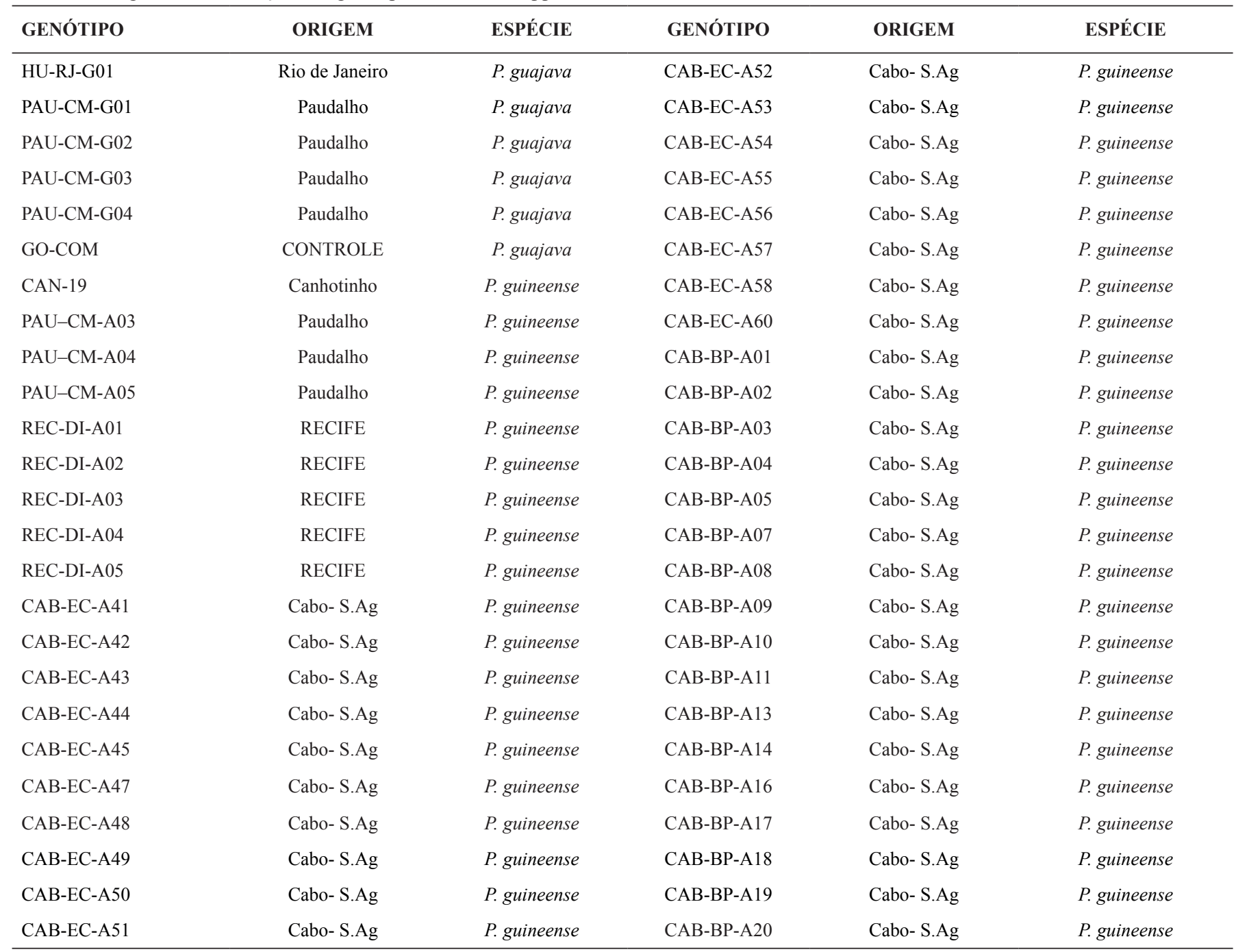


MAP e 1,25 g de Ultraferro $^{\circledR}$, dissolvidos em $50 \mathrm{~L}$ de água, a cada 15 dias. Quando as mudas alcançaram $8 \mathrm{~cm}$ de altura foram transplantas para sacos plásticos de $20 \mathrm{~cm}$ de altura por $10 \mathrm{~cm}$ de largura, com substrato comercial Basaplant $^{\circledR}$, devidamente identificado para cada genótipo e acondicionadas em casa de vegetação onde receberam os devidos tratos culturais, irrigação por aspersão e solução nutritiva. O delineamento utilizado foi inteiramente casualizado, correspondendo aos 44 genótipos de araçazeiros, e seis genótipos de goiabeiras. Cada tratamento continha seis repetições, onde cada parcela foi constituída por uma planta.

O inóculo, cedido pela Embrapa Semiárido - CPATSA-Petrolina-PE, foi propagado em tomateiro (Solanum lycopersicum L.) reconhecido como bom hospedeiro ao M. enterolobii. Para a extração de ovos seguiuse a metodologia de Hussey \& Barker (17). A contagem de ovos foi feita com uso de microscópio ótico e de câmera de contagem de Peters, usando-se alíquota de $1 \mathrm{~mL}$ em $9 \mathrm{~mL}$ de água destilada para ajuste de $1000 \mathrm{ovos} / \mathrm{ml}$. Transcorrido 60 dias após o transplantio das mudas para os sacos plásticos, os genótipos foram inoculados com M. enterolobii com suspensão aquosa contendo 8.000 ovos por planta. Juvenis de segundo estádio não foram incluídos na contagem. Após a inoculação foi suspensa a irrigação por período de 24 horas, a fim e evitar a lixiviação de ovos no perfil do solo.

Aos 120 dias após a inoculação foram avaliados: índice de galhas (IG), obtido por meio da escala de notas do International Meloidogyne Project (IMP), utilizado por Taylor \& Sasser (32), o número de ovos por grama de raiz (OGR) e o fator de reprodução (FR). As reações dos hospedeiros foram enquadradas nos parâmetros estabelecidos por Hartman \& Sasser (16). Plantas que apresentaram valores de IG $\leq 2$ foram consideradas resistentes. A estimativa do número de ovos por sistema radicular foi realizada com auxílio de câmara de Peters, em microscópio ótico, obtendo-se assim a população final (Pf).
O fator de reprodução (FR) obtido pelo quociente $\mathrm{Pf} / \mathrm{Pi}$, estabeleceu as reações da espécie vegetal quanto ao parasitismo, sendo enquadrados nos parâmetros estabelecidos por Oostenbrink (25), onde FR $<1$ espécie resistente (R), FR $=0$ espécie imune (I) e FR $>1$ espécie suscetível (S). $\mathrm{O}$ fator de reprodução foi comparado pelo teste de Scott-Knot a 5\% de probabilidade. O maior valor obtido para a variável FR foi usado como referência para o cálculo da redução do fator de reprodução (RFR) segundo a metodologia de Moura \& Régis (24) e classificados nas seguintes faixas: 0 a $25=$ planta altamente suscetível; 26 a $50=$ planta suscetível; 51 a $75=$ planta pouco resistente; 76 a $95=$ planta moderadamente resistente; 96 a 99 = planta resistente; 100 = planta imune.

\section{RESULTADOS E DISCUSSÃO}

Durante os 120 dias do período de infecção as temperaturas observadas em casa de vegetação variaram entre $25,5^{\circ} \mathrm{C}$ e $39^{\circ} \mathrm{C}$. Após este período, observou-se a presença de galhas nas raízes das plantas inoculadas sugerindo que o ciclo de reprodução do patógeno se completou (Figura 1A). De acordo com Agrios (1), o ciclo de vida das espécies do gênero Meloidogyne pode variar de acordo com o seu hospedeiro e a temperatura ambiente, durando aproximadamente 25 dias a $27^{\circ} \mathrm{C}$, se alongando em temperaturas mais baixas ou mais elevadas. A partir de dois meses da inoculação das plantas com M. enterolobii, sintomas de parasitismo como murcha em horários mais quentes do dia, amarelecimento das folhas e bronzeamento foram observados em alguns dos genótipos inoculados. Também foi possível observar sintomas como engrossamento de raízes e redução do crescimento (Figuras 1B e 1C). No extrato de raízes dos genótipos inoculados foram observados a presença de ovos e J2 (Figura 1D).

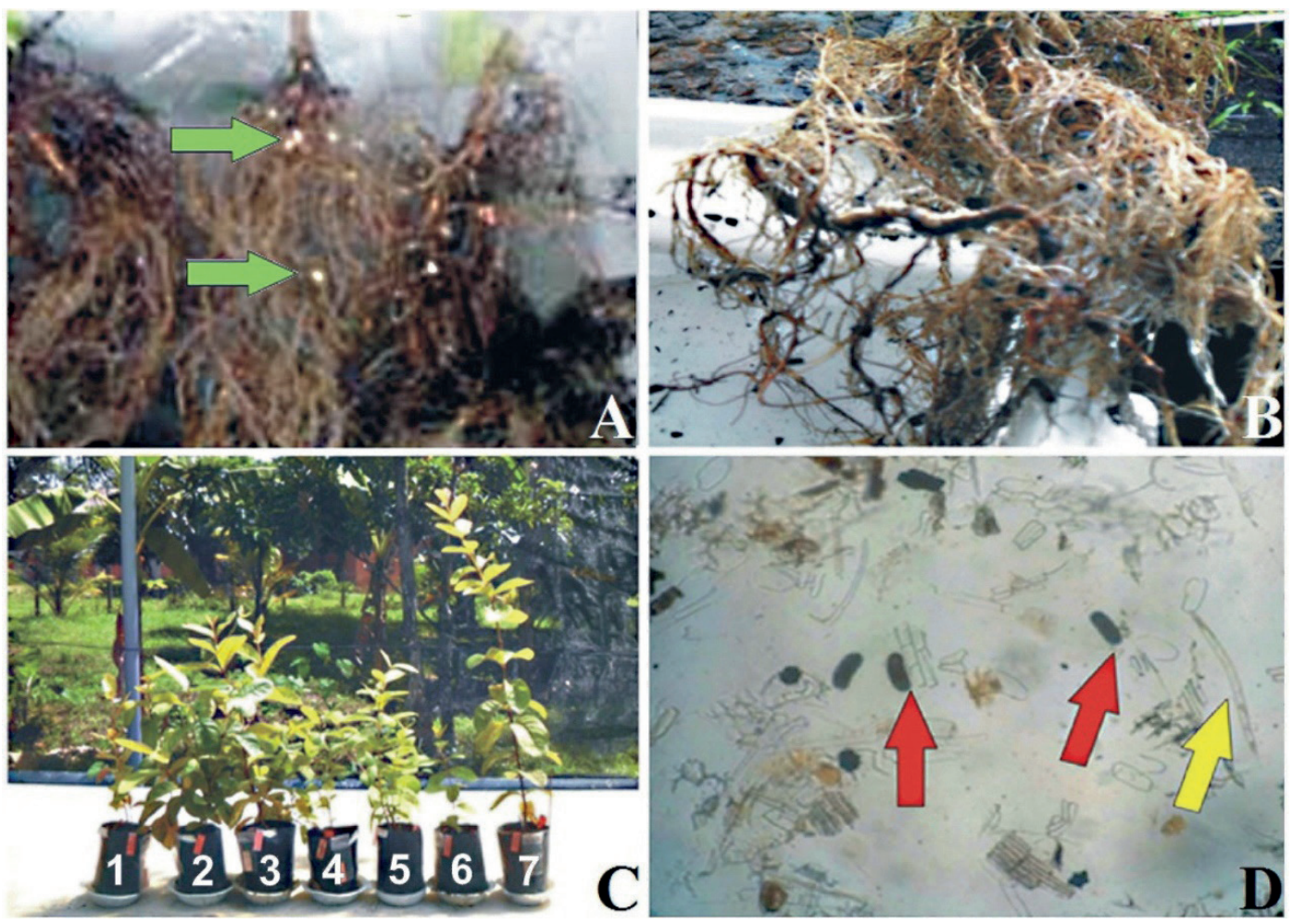

Figura 1. Sintomas causados por Meloidogyne enterolobii Yang e Eisenback. Setas verdes indicam a presença de galhas nas raízes dos genótipos araçazeiro (A), Engrossamento de raízes na goiabeira (B), Redução de crescimento do genótipos de araçazeiro 1, 2, 3, 4, 5 e 6, em contraste com o genótipo 7 não inoculado (C) e presença de ovos (setas vermelhas) e juvenis de segundo estádio, J2, (seta amarela) no extrato de raízes dos genótipos inoculados (D). 
$\mathrm{O}$ fator de reprodução calculado para cada genótipo variou entre 0,18 (REC-DI-A04) e 9,22 (CAB-BP-A19) (Tabela 2), evidenciando ampla variabilidade na resposta dos genótipos ao parasitismo do nematoide. Dentre os parâmetros utilizados para medição da resposta ao parasitismo do nematoideos, o FR é o mais preciso, sendo sensível a alteração de quantidade de inóculo e período anual de inoculação para quantificar o nível de resistência na interação planta-nematoide, fato este também observado por Burla et al. (4).

Todos os genótipos oriundos da população REC-DI, e o genótipo PAU-CM-A03 apresentaram valores de FR $<1$, indicando sua resistência ao patógeno. Todos os demais genótipos foram classificados como suscetíveis $(\mathrm{FR}>1)$. O grau de suscetibilidade dos genótipos avaliados variou grandemente, com genótipos com valores de FR ligeiramente acima de 1, o que caracteriza uma baixa multiplicação do parasita, e valores de FR próximos de 10, indicando uma extrema suscetibilidade à multiplicação de $M$. enterolobii. Entre os genótipos de goiabeira avaliados, HU-RJ-G01 e PAU-CM-G03, apresentaram valores de FR de 1,43 e 1,69, respectivamente, o que contrasta com o observado na literatura, onde goiabeiras são relatadas como bastante suscetíveis a este parasita, com valores de FR variando entre 5,6 (30) e 42,81 (22).

Entre os araçazeiros com FR pouco acima de 1, o genótipo CAB-EC-A50 apresentou o valor de 1,20, sendo classificado como moderadamente resistente. Desta forma, estes genótipos por apresentarem fator de reprodução pouco acima de 1, poderão ser utilizados em futuros estudos de resistência ao M. enterolobii. A partir do RFR que compara os valores de FR de cada genótipo com o valor de FR do genótipo mais afetado (CAB-BP-A19), segundo a metodologia de Moura \& Régis (24), os genótipos foram classificados de acordo com sua reação ao fitoparasita. Segundo este critério, os genótipos RECDI-A02 e REC-DI-A04 foram os únicos classificados como resistentes, sendo os mais promissores para futuros estudos de compatibilidade para uso como porta-enxerto de goiabeiras comerciais.

Em relação ao IG, observou-se uma variação de 2,16 do genótipo REC-DI-A02 a 4,66 do genótipo CAB-EC-A44 (Tabela 2). Baseado neste critério, os genótipos REC-DI-A02, REC-DI-A04, REC-DI-A05 são considerados resistentes. No entanto, apenas a observação deste critério pode levar a classificações errôneas, devido à sua subjetividade

Tabela 2. Avaliação da patogenicidade de Meloidogyne enterolobii Yang e Eisenback em araçazeiros e goiabeiras da região da Zona da Mata do Estado de Pernambuco. UFRPE, Recife-PE, 2017

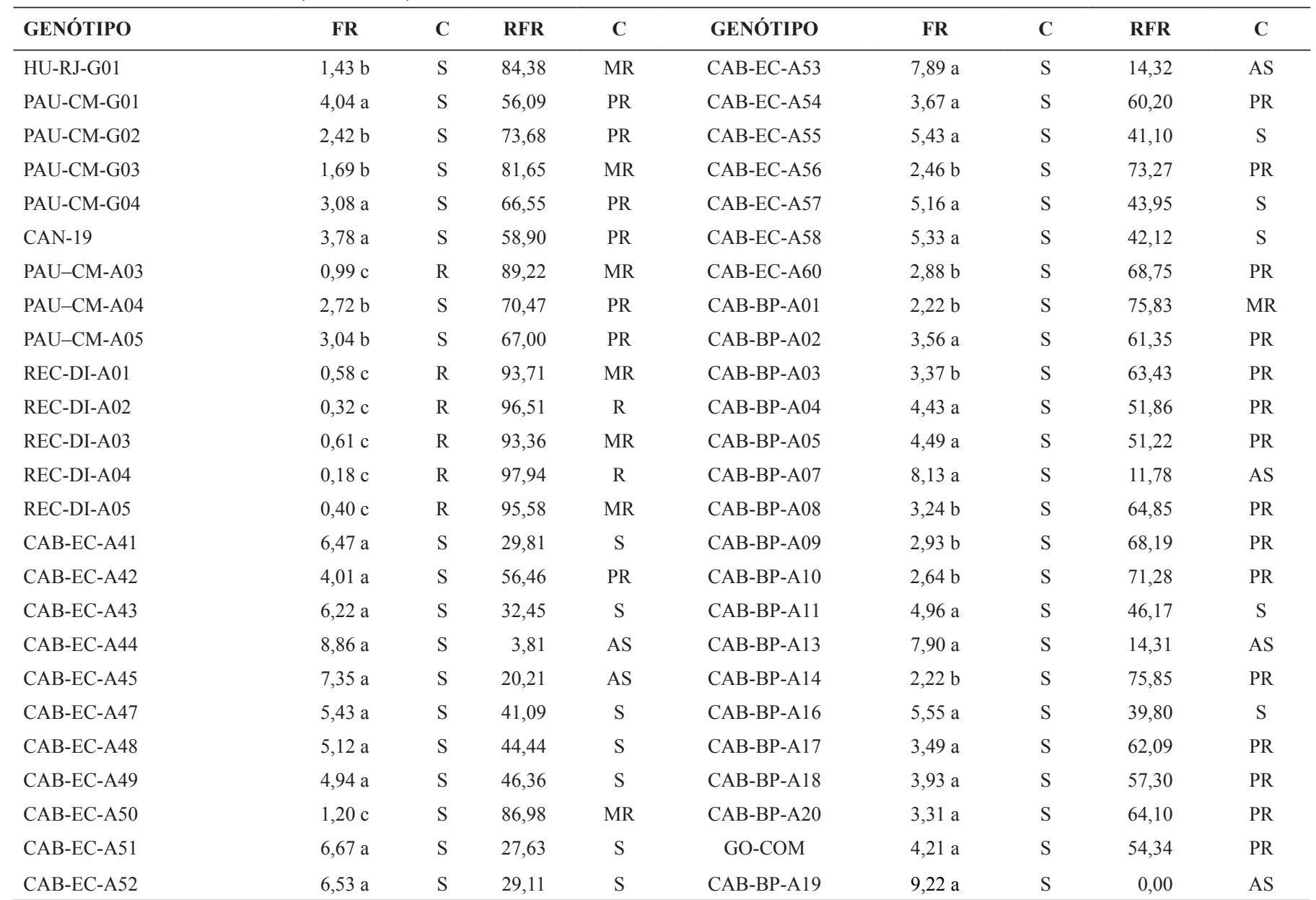

Médias seguidas pela mesma letra não diferem estatisticamente entre si pelo Teste de Scott-Knott ao nível de 5\% de probabilidade. FR - Fator de Reprodução, RFR - Redução do Fator de Reprodução. Em que: $\mathrm{FR}<1$ espécie resistente (R), $\mathrm{FR}=0$ espécie imune (I) e FR>1 espécie suscetível (S). C - Classificação: R (Resistente), S (Suscetível), AS (Altamente Suscetível), PR (Pouco Resistente), MR (Moderadamente Resistente). FR = População Final/População Inicial, PF = Quantidade de ovos e J2 observados ao final, a partir dos 8000 ovos iniciais de inóculo (22). Letras ao lado dos valores de FR referem-se à análise de agrupamento pelo teste de Scott-Knot (5\%) dos valores de FR transformados para raiz de x, utilizou-se o software Assistat 7.7. 
e empirismo, de acordo com Costa Filho (9). A análise de correlação entre as variáveis FR e IG revelou uma correlação positiva entre os dois índices $(\mathrm{R}=0,605)$, indicando que existe uma tendência de que genótipos exibindo valores elevados de IG também apresentem valores elevados de FR (Figura 2).

Os dados de OGR e PF demonstraram grande variação entre os genótipos analisados (Tabela 3), de forma similar ao observado pela análise do FR, os genótipos da população REC-DI apresentaram os resultados mais promissores, diferindo estatisticamente dos demais genótipos.

Diversos estudos sobre a reação de genótipos e variedades comerciais de goiabeira ao parasitismo de $M$. enterolobii, demonstraram sua extrema suscetibilidade a este parasita $(22,27,30)$. As espécies da família Myrtaceae apresentam diferentes níveis de resistência/ suscetibilidade ao parasitismo de $M$. enterolobii. Apesar de genótipos imunes terem sido encontrados em espécies do gênero Psidium, como $P$. cattleyanum, $P$. myrtoides e $P$. acutangulum, as espécies $P$. guajava $e P$. guineense são consideradas mais suscetíveis a este fitonematóide (21). Apesar da resistência encontrada em $P$. cattleyanum, testes de enxertia indicaram uma incompatibilidade para ser utilizada como porta-enxerto com a cultivar Paluma (2). Desta forma é de grande importância, a busca de genótipos resistentes ao parasita, mas também que tenham maior possibilidade de compatibilidade com variedades comerciais de goiabeira (10).

Os genótipos de araçazeiros REC-DI-A02 e REC-DI-A04 por terem alcançado os melhores resultados na avaliação de redução do FR, podem ser indicados como fonte de resistência a M. enterolobii e também como genótipos promissores para testes de enxertia com variedades comerciais de goiabeira.

\section{AGRADECIMENTOS}

O presente trabalho foi desenvolvido com recursos do Programa de Pós Graduação em Melhoramento Genético de Plantas e da Coordenação de Aperfeiçoamento de Pessoal de Nível Superior (CAPES). Agradecemos também a CAPES pela concessão das bolsas de Mestrado e Pós Doutorado à Edilton de A. Cavalcanti Junior e Rômulo M. Moraes Filho, respectivamente.

Tabela 3. Reação de genótipos das espécies $P$. guajava e $P$. guineense ao parasitismo de $M$. enterolobii.

\begin{tabular}{|c|c|c|c|c|c|c|c|}
\hline GENÓTIPO & IG & OGR $^{*}$ & $\mathbf{P F}^{*}$ & GENÓTIPO & IG & OGR $^{*}$ & $\mathbf{P F}^{*}$ \\
\hline PAU-CM-G01 & $4,50 \mathrm{a}$ & $39,14 \mathrm{~b}$ & $176,86 \mathrm{a}$ & CAB-EC-A54 & $3,66 \mathrm{a}$ & $41,61 \mathrm{~b}$ & $169,24 \mathrm{a}$ \\
\hline PAU-CM-G03 & $4,33 \mathrm{a}$ & $25,44 \mathrm{c}$ & $113,42 \mathrm{~b}$ & CAB-EC-A56 & $3,00 \mathrm{~b}$ & $30,97 \mathrm{c}$ & $128,09 \mathrm{~b}$ \\
\hline PAU-CM-G04 & $4,66 \mathrm{a}$ & $27,20 \mathrm{c}$ & $155,34 \mathrm{a}$ & CAB-EC-A57 & $4,50 \mathrm{a}$ & $41,80 \mathrm{~b}$ & $195,54 \mathrm{a}$ \\
\hline PAU-CM-A03 & $3,66 \mathrm{a}$ & $24,79 \mathrm{c}$ & $84,66 \mathrm{c}$ & CAB-EC-A60 & $3,50 \mathrm{~b}$ & $59,20 \mathrm{a}$ & $147,31 \mathrm{~b}$ \\
\hline PAU-CM-A04 & $4,16 \mathrm{a}$ & $42,92 \mathrm{~b}$ & $143,89 \mathrm{~b}$ & CAB-BP-A01 & $4,33 \mathrm{a}$ & $33,34 \mathrm{c}$ & $129,56 \mathrm{~b}$ \\
\hline PAU-CM-A05 & $4,16 \mathrm{a}$ & $38,39 \mathrm{~b}$ & $137,91 \mathrm{~b}$ & CAB-BP-A02 & $4,33 \mathrm{a}$ & $36,36 \mathrm{~b}$ & $160,33 \mathrm{a}$ \\
\hline REC-DI-A01 & $3,16 \mathrm{~b}$ & $15,90 \mathrm{~d}$ & $64,80 \mathrm{c}$ & CAB-BP-A03 & $4,16 \mathrm{a}$ & $28,04 \mathrm{c}$ & $146,02 \mathrm{~b}$ \\
\hline REC-DI-A02 & $2,16 \mathrm{c}$ & $13,39 \mathrm{~d}$ & $49,53 \mathrm{c}$ & CAB-BP-A04 & $4,50 \mathrm{a}$ & $39,71 \mathrm{~b}$ & $179,65 \mathrm{a}$ \\
\hline CAB-EC-A41 & $4,16 \mathrm{a}$ & $40,42 \mathrm{~b}$ & $213,29 \mathrm{a}$ & CAB-BP-A09 & $4,50 \mathrm{a}$ & $36,93 \mathrm{~b}$ & $150,93 \mathrm{~b}$ \\
\hline CAB-EC-A42 & $4,16 \mathrm{a}$ & $39,15 \mathrm{~b}$ & $174,80 \mathrm{a}$ & CAB-BP-A10 & $4,16 \mathrm{a}$ & $32,87 \mathrm{c}$ & $138,65 \mathrm{~b}$ \\
\hline CAB-EC-A43 & $4,16 \mathrm{a}$ & $38,57 \mathrm{~b}$ & 209,97 a & CAB-BP-A11 & $4,50 \mathrm{a}$ & 48,19 a & $190,46 \mathrm{a}$ \\
\hline CAB-EC-A44 & $4,66 \mathrm{a}$ & $45,77 \mathrm{~b}$ & $260,49 \mathrm{a}$ & CAB-BP-A13 & $4,33 \mathrm{a}$ & $44,20 \mathrm{~b}$ & $225,30 \mathrm{a}$ \\
\hline CAB-EC-A45 & $4,33 \mathrm{a}$ & $46,85 \mathrm{a}$ & $228,18 \mathrm{a}$ & CAB-BP-A14 & $3,83 \mathrm{a}$ & $37,11 \mathrm{~b}$ & $128,29 \mathrm{~b}$ \\
\hline CAB-EC-A47 & $3,66 \mathrm{a}$ & $52,57 \mathrm{a}$ & $193,61 \mathrm{a}$ & CAB-BP-A16 & $3,66 \mathrm{a}$ & $40,49 \mathrm{~b}$ & $196,27 \mathrm{a}$ \\
\hline CAB-EC-A48 & $4,00 \mathrm{a}$ & $36,19 \mathrm{~b}$ & 189,46 a & CAB-BP-A17 & $3,83 \mathrm{a}$ & $27,72 \mathrm{c}$ & $155,52 \mathrm{a}$ \\
\hline CAB-EC-A49 & $3,83 \mathrm{a}$ & $29,91 \mathrm{c}$ & $185,80 \mathrm{a}$ & CAB-BP-A18 & $4,16 \mathrm{a}$ & $34,90 \mathrm{~b}$ & $164,81 \mathrm{a}$ \\
\hline CAB-EC-A50 & $3,50 \mathrm{~b}$ & $18,35 \mathrm{~d}$ & $95,10 \mathrm{c}$ & CAB-BP-A20 & $4,16 \mathrm{a}$ & $34,70 \mathrm{~b}$ & $159,66 \mathrm{a}$ \\
\hline
\end{tabular}

Médias seguidas pela mesma letra não diferem estatisticamente entre si pelo Teste de Scott-Knott ao nível de 5\% de probabilidade. IG - Índice de galhas, OGR Ovos por grama de raiz (g), PF = População Final, * = Dados Transformados para Raiz de X. 

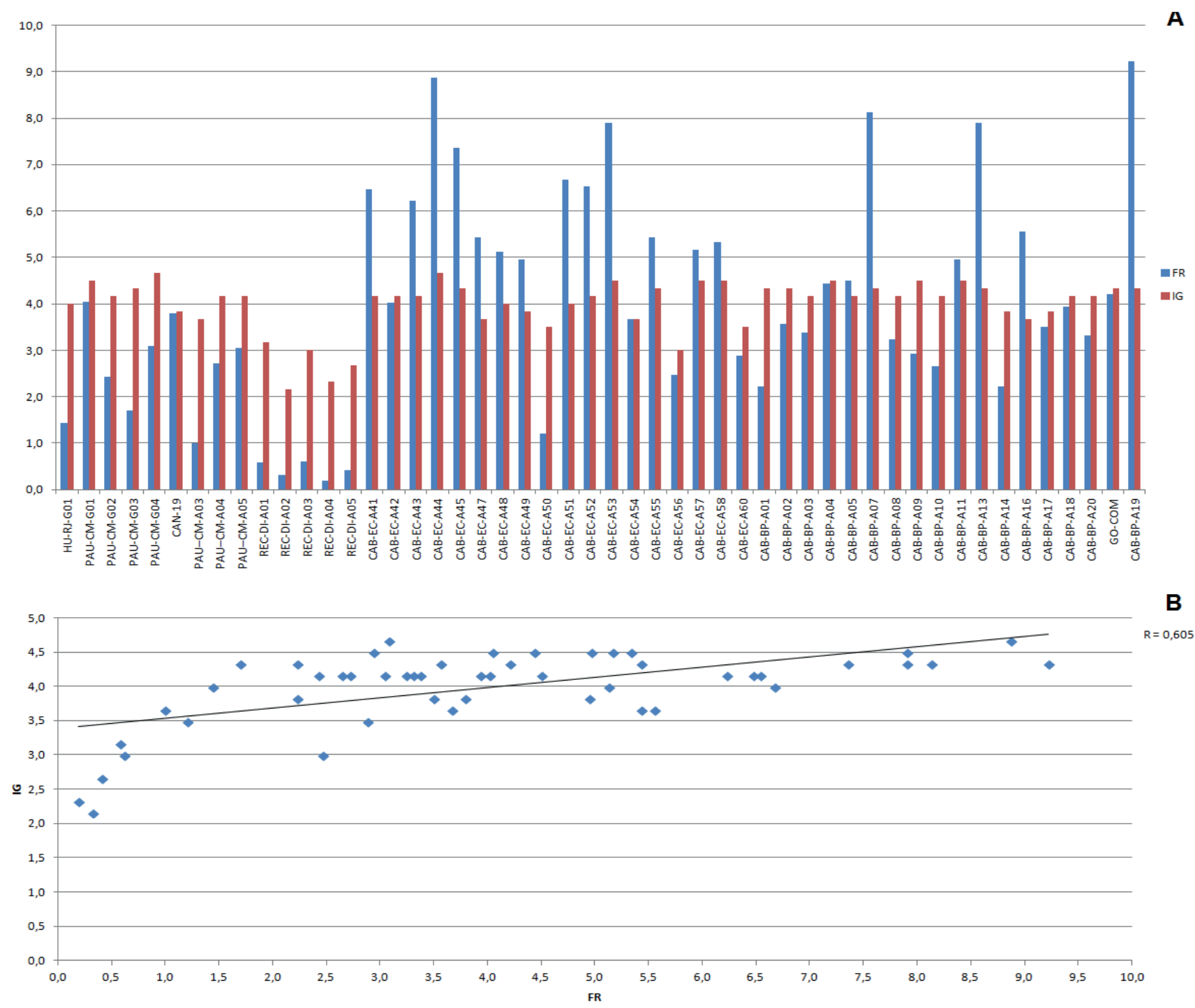

Figura 2. Índice de galhas (IG) e fator de reprodução (FR) de $M$. enterolobii em genótipos de araçazeiro e goiabeira (A) e correlação entre FR e IG (B).

\section{REFERÊNCIAS}

1. Agrios, G.N. Plant pathology. 5.ed. New York: Elsevier Academic Press, 2005. 952p

2. Almeida, E.J.; Soares, P.L.M.; Santos, J.M.; Martins, A.B.G. Resistência de goiabeiras e araçazeiros a Meloidogyne mayaguensis. Pesquisa Agropecuária Brasileira, Brasília, DF, v.44, n.4, p.421-423, 2009.

3. Biazatti, M.A.; Souza, R.M.; Marinho, C.S.; Guilherme, D.O.; Campos, G.S.; Gomes, V.M.; Bremenkamp, A.C. Resistência de genótipos de araçazeiros a Meloidogyne enterolobii. Ciência Rural, Santa Maria, v.46, n.3, p.418-420, 2016.

4. Burla, R.S.; Souza, R.M.; Gomes, V.M.; Corrêa, F.M. Comparação entre níveis de inóculo, épocas de avaliação e variáveis para seleção de Psidium spp. visando à resistência a Meloidogyne mayaguensis. Nematologia Brasileira, Brasília, DF, v.34, n.2, p.82-90, 2010.

5. Carneiro, R.M.D.G.; Moreira, W.A.; Almeida, M.R.A.; Gomes, A.C.M.M. Primeiro registro de Meloidogyne mayaguensis em goiabeira no Brasil. Nematologia Brasileira, Brasília, DF, v.25, n.2, p.223-228, 2001.

6. Carneiro, R.G.; Monaco, A.P.A.; Moritz, M.P.; Nakamura, K.C.; Scherer, A. Identificação de Meloidogyne mayaguensis em goiabeira e em plantas invasoras, em solo argiloso, no Estado do Paraná. Nematologia Brasileira,
Brasília, DF, v.30, n.3, p.293-298, 2006.

7. Carneiro, R.M.D.G.; Siqueira, K.M.S.; Santos, M.F.A.; Almeida, M.R.A.; Tigano, M.S. Ocorrência de Meloidogyne mayaguensis em goiabeira e mamoeiro no estado de Goiás. Fitopatologia Brasileira, Brasília, DF, v.33, p.258, 2008. Suplemento.

8. Charchar, J.M.; Fonseca, M.E.N.; Boiteux, L.B.; Lima Neto, A.F. Ocorrência de Meloidogyne mayaguensis em goiabeira no Estado de Tocantins. Nematologia Brasileira, Piracicaba, v.33, n.2, p.182-186, 2009.

9. COSTA FILHO, J.H.; Queiroz, M.A.; Castro, J.M.C.; Fontes, L.A.; Preston, H.A.F.; Santos, T.S.; Carvalho, N.F.O.; Silva, S.C.A.; Santos, M.F.; Candido, D. Reaction of watermelon accessions to Meloidogyne enterolobii. African journal of agricultural research, Abuja, v. 13, p. 1948-1953, 2018.

10. Freitas, V.M.; Correa, V.R.; Motta, F.C.; Sousa, M.G.; Gomes, A.C.M.M.; Carneiro, M.D.G.; Silva, D.B.; Mattos, J.K.; Nicole, M.; Carneiro, R.M.D.G. Resistant accessions of wild Psidium spp. to Meloidogyne enterolobii and histological characterization of resistance. Plant Pathology, London, v.63, n.4, p.738-746, 2014.

11. Gomes, V. M. Meloidoginose da goiabeira: estudos sobre a sua patogênese e formas de convívio com a doença a campo. Dissertação - Universidade Estadual do Norte Fluminense Darcy Ribeiro, Campos dos Goytacazes (RJ), 2007.

12. Gomes, C.B.; Couto, M.E.O.; Carneiro, R.M.D.G. Registro de ocorrência 
de Meloidogyne mayaguensis em goiabeira e fumo no Sul do Brasil. Nematologia Brasileira, Piracicaba, v.32, n.3, p.244-247, 2008.

13. Gomes, V.M.; Souza, R.M., Mussi-Dias, V.; Silveira, S.F.; Dolinski, C. Guava decline: a complex disease involving Meloidogyne mayaguensis and Fusarium solani. Journal of Phytopathology, Nova Jersey, v.158, p.45-50, 2011.

14. Gonzaga Neto, L. Produção de goiaba. Fortaleza: Instituto Frutal, 2007. 64p.

15. Guimarães, L.M.P.; Moura, R.M.; Pedrosa, E.M.R. Parasitismo de $\mathrm{Me}$ loidogyne mayaguensis em diferentes espécies botânicas. Nematologia Brasileira, Piracicaba, v.27, n.2, p.139-147, 2003.

16. Hartman, K.M.; Sasser, J.N. Identification of Meloidogyne species on the basis of differential host test and perineal pattern morphology. In: Sasser, J.N.; Carter, C.C.; Barker, K.R. An advanced treatise on meloidogyne: methodology. Raleigh: North Carolina State University Graphics, 1985. v.2, p.69-77.

17. Hussey, R.S.; Barker, K.R. A Comparison of methods of collecting inocula of Meloidogyne spp. including a new technique. Plant Disease Reporter, Saint Paul, v.57, p.1025-1028, 1973.

18. IBGE - INSTITUTO BRASILEIRO DE GEOGRAFIA E ESTATÍSTICA. Produção Agrícola Municipal. Rio de Janeiro, 2019. Disponível em: https://sidra.ibge.gov.br/tabela/5457.

19. LIMA, I. M.; MARTINS, M. V. V.; SERRANO, L. A. L.; CARNEIRO, R. M. D. G. Ocorrência de Meloidogyne mayaguensis em goiabeira cv. 'Paluma' no estado do Espírito Santo. Resumo. In: XXVII CONGRESSO BRASILEIRO DE NEMATOLOGIA, 2007, Goiânia. Anais... Goiânia: UFG, p. 96-97, 2007.

20. Maranhão, S.R.V.L.; Moura, R.M.; Pedroza, E.M.R. Reação de indivíduos segregantes de goiabeira a Meloidogyne incógnita raça1 e M. mayaguensis. Nematologia Brasileira, Piracicaba, v.25, n.2, p.191-195, 2001.

21. Marques, M.L.S.; Pimentel, J.P.; Tavares, O.C.H.; Veiga, C.F.M.; Berbara, R.L.L. Hospedabilidade de diferentes espécies de plantas a meloidogyne enterolobii no Estado do Rio de Janeiro. Nematropica, Auburn, v.42, n.2, p.303-313, 2012.

22. Martins, L.S.S.; Musser, R.S.; Souza, A.G.; Resende, L.V.; Maluf, W.R. Parasitismo de Meloidogyne enterolobii em espécies de Myrtaceae. Revista Brasileira de Fruticultura, Jaboticabal, v.35, n.2, p.477-484, 2013.
23. Moreira, A.A.; Martins, L.S.S.; Musser, R.S.; Moraes Filho, R.S.; Maranhão, W.; Rossiter, J.G.; Montarroyos, A.V.V. Response of Malpighia emarginata active germplasm bank accessions to Meloidogyne enterolobii parasitism. Genetics and Molecular Research, Ribeirão Preto, v.15, n.4, gmr. 15048868, 2016.

24. Moura, R.M.; Régis, E.M.O. Reações de cultivares de feijoeiro comum (Phaseolus vulgaris) em relação ao parasitismo de Meloidogyne javanica e M. incognita (Nematoda: Heteroderidae). Nematologia Brasilileira, Piracicaba, v.11, p.215-225, 1987.

25. Oostenbrink, M. Major characteristics of the relation between nematodes and plants. Med Van De landbouwhogeschool, Wageningen, v.66, n.4, p.46, 1966.

26. Pereira, F.M.; Kavati, R. Contribuição da pesquisa científica brasileira no desenvolvimento de algumas frutíferas de clima subtropical. Revista Brasileira de Fruticultura, Jaboticabal, v.33, p.92-108, 2011.

27. Pereira, K.C.; Soares, P.L.M.; Santos, J.M.; Batista, E.S.P.; Maldonato Jr, W.J. Desenvolvimento de cultivares de goiabeira inoculadas com Meloidogyne enterolobii. Nematropica, Auburn, v.46, n.1, p.54-59, 2016.

28. Raseira, M.C.B.; Raseira, A. Contribuição ao estudo do araçazeiro, Psidium cattleyanum. Pelotas: Embrapa-CPACT, 1996. v.1, 95p.

29. Rodríguez, N.N.; Valdés-Infante, J.J.; Rodríguez, J.A.; Velásquez, J.B Genetic resources and breeding of guava (Psidium guajava L.) in Cuba. Biotecnologia Aplicada, Cubanacán, v.27, n.3, p.238-241, 2010.

30. Scherer, A.; Carneiro, R.G.; Mônaco, A.P.A.; Moritz, M.P.; Nakamura, K.C.; Gomes, J.C.; Torrezani, N.C.; Santiago, D.C.; Carneiro, R.M.D.G. Reação de Genótipos de Psidium guajava a Meloidogyne enterolobii. Nematologia Brasileira, Piracicaba, v.36, n.1/2, p.50-53, 2012.

31. Souza, R.M.; Nogueira, M.S.; Lima, I. M.; Melarato, M.; Dolinski, C.M Manejo de nematoides das galhas da goiabeira em São João da Barra (RJ) e relato de novos hospedeiros. Nematologia Brasileira, Piracicaba, v.30, n.2, p.165-169, 2006.

32. Taylor, A.L.; Sasser, J.N. Biología, identificación y control de los nematodos del nódulo de la raíz (Espécies de Meloidogyne). Raleigh: Universidad del Estado de Carolina del Norte, 1983. 111p.

33. Yang B.; J. D. Eisenback, J.D. (1983). "Meloidogyne enterolobii n. sp. (Meloidogynidae), a root-knot nematode parasitizing pacara ear pod tree in China". Journal of Nematology. Nova Jersey, v. 15 n.3, p. 381-391, 1983. 